(2) Open Access Full Text Article

REVIEW

\title{
Improving Exercise-Based Interventions for People Living with Both COPD and Frailty: A Realist Review
}

This article was published in the following Dove Press journal:

International Journal of Chronic Obstructive Pulmonary Disease

\author{
Lisa Jane Brighton (1D \\ Catherine J Evans $\mathbb{D}^{1,2}$ \\ William DC Man $\mathbb{D}^{3,4}$ \\ Matthew Maddocks (D) \\ 'Cicely Saunders Institute of Palliative \\ Care, Policy and Rehabilitation, King's \\ College London, London, UK; ${ }^{2}$ Brighton \\ General Hospital, Sussex Community \\ NHS Foundation Trust, Brighton, UK; \\ ${ }^{3}$ National Heart and Lung Institute, \\ Imperial College, London, UK; ${ }^{4}$ Harefield \\ Respiratory Research Group, Royal \\ Brompton and Harefield NHS Foundation \\ Trust, London, UK
}

Background: People living with both chronic obstructive pulmonary disease (COPD) and frailty have high potential to benefit from exercise-based interventions, including pulmonary rehabilitation, but face challenges completing them. Research to understand ways to optimise exercise-based interventions in this group is lacking. We aimed to understand how exercisebased interventions might improve outcomes for people living with both COPD and frailty.

Methods: This realist review used database searches and handsearching until October 2019 to identify articles of relevance to exercise-based interventions for people living with COPD and frailty. A scoping search explored what is important about the context of living with COPD and frailty, and what mechanisms might be important in how exercise-based interventions result in their intended outcomes. Through discussion with stakeholders, the review scope was refined to areas deemed pertinent to improving care. We retained articles within this refined scope and identified additional articles through targeted handsearching. Data were extracted and synthesised in a narrative, prioritised by relevance and rigour.

Results: Of 344 records identified, 35 were included in the review and 20 informed the final synthesis. Important contextual factors to consider included: negative beliefs about themselves and exercise-based interventions; heterogenous presentation and comorbidities; decreased reserves and multidimensional loss; and experiencing unpredictable health and disruptions. In these circumstances, mechanisms that may help maximise outcomes from exercise-based interventions included: trusting relationships; creating a shared understanding of needs; having the capacity to address multidimensional concerns; being able to individualise approaches to needs and priorities; and flexible approaches to intervention delivery. Mixed-methods research and explicit theorising were often absent.

Conclusion: Building trusting relationships, understanding priorities, using individualised and multidisciplinary approaches, and flexible service delivery can improve the value of exercise-based interventions for people living with both COPD and frailty. Development and evaluation of new and adapted interventions should consider these principles.

Keywords: chronic obstructive pulmonary disease, frailty, rehabilitation, exercise, review

\section{Plain Language Summary}

Why did we do this review? Exercise-based services can improve the health of people with both chronic obstructive pulmonary disease (COPD) and frailty. However, these individuals can find participation challenging. We wanted to understand how and why some exercise-based interventions work better than others for people with COPD and frailty, to improve future care.

How did we do this review? We searched scientific databases and drew on other sources (e.g. expert recommendations) to find articles about exercise-based
Correspondence: Lisa Jane Brighton King's College London, Cicely Saunders Institute of Palliative Care, Policy and

Rehabilitation, London, UK

Tel +44207848504l

Email lisa.brighton@kcl.ac.uk 
interventions for people living with both COPD and frailty. We worked with professionals and service users to ensure the review focused on their priorities for future care. We focused on the higher quality and most relevant studies in our summary.

What did we find? People with both COPD and frailty tend to hold negative views of themselves and services; live with multiple health conditions, and experience unpredictable and poor physical, emotional and social health. Exercise-based interventions for this group should consider building trusting relationships; creating a shared understanding of needs; and be able to individualise approaches to needs and priorities. Interventions should also be able to address physical, emotional and social concerns; and take more flexible approaches in their delivery.

\section{Introduction}

Approximately one in five people with COPD live with frailty, and over half are considered pre-frail. ${ }^{1}$ Frailty is broadly understood as a multidimensional syndrome characterised by decreases in reserve and diminished resistance to stressors. ${ }^{2}$ Within this broader understanding lies the concept of physical frailty: reduced physiological and physical function that increases a person's risk of dependency and/or death. ${ }^{3}$ The mechanisms underlying the link between COPD and frailty are likely multifactorial. Examples could include sedentary behaviour, mobility impairments, sarcopenia and malnutrition, in addition to underlying demographic risk factors such as age and socioeconomic status. ${ }^{1,4}$

People with both COPD and frailty are at increased risk of mortality ${ }^{5,6}$ and readmission within 3-months posthospitalisation for an exacerbation of their disease. ${ }^{7,8}$ Compared to people who have COPD but no indication of frailty, they experience poorer physical function and health status, ${ }^{9}$ and increased anxiety and depression symptoms. ${ }^{10}$ People with COPD and frailty are also less likely to start and complete pulmonary rehabilitation, ${ }^{10}$ and have lower levels of physical activity. ${ }^{11}$

Supporting engagement with exercise-based interventions is a priority for people with COPD and frailty. ${ }^{12}$ Participating in physical activity and exercise is known to improve outcomes for people with COPD ${ }^{13-15}$ and with frailty. ${ }^{16-18}$ People living with both COPD and frailty can experience improved breathlessness, exercise performance, physical activity levels and health status when they complete exercise-based interventions. ${ }^{10,19}$ However, there is an accompanying body of literature recognising that people with $\mathrm{COPD}^{20-23}$ and frailty ${ }^{24,25}$ face challenges to participating in physical activity.

Engagement in exercise-based interventions is inherently complex: it involves multiple interacting components, often relies on complex behaviours, can require a degree of flexibility and tailoring and have multiple, varying affects. ${ }^{26}$ Moreover, exercise-based interventions occur in complex systems, made up of individual, organisational and societal influences. $^{27,28}$ How and why some exercise-based approaches are more effective than others in different circumstances is not understood. Understanding key contextual factors and mechanisms underlying successful interventions is therefore required to create transferrable knowledge for future developments. ${ }^{28,29}$

We aimed to understand how exercise-based interventions might improve outcomes for people living with both COPD and frailty, in order to give practical recommendations for future clinical practice and research.

\section{Methods \\ Design}

We completed a realist review drawing on published guidance, ${ }^{30}$ and registered the protocol on PROSPERO to aid transparency. Realist reviews have been used to understand a variety of complex health interventions with multimorbid populations. ${ }^{31,32}$ The methods draw on a realist philosophy, taking a "generative" view of how interventions work (including contextual factors), as opposed to a more positivist "successionist" view. ${ }^{33}$ Realist reviews are explanatory, seeking to unpick the important mechanisms underlying sets of interventions. This approach allows researchers to move beyond individual studies, and focus on the main ideas or theories on which they are based. ${ }^{34}$ In this review, we consider exercise-based interventions for people with COPD, and the mechanisms that might be important when applying them in the context of also living with frailty.

Key stages of the review are outlined below and in Figure 1. In some instances, in line with a realist approach, we moved back and forth between these stages iteratively as learning progressed. We involved relevant stakeholders throughout, including professionals in respiratory care, general practice, geriatrics, and pulmonary rehabilitation, respiratory researchers, and people with personal experience relevant to living with COPD and frailty. We invited stakeholders to help us shape the focus of the review, and 


\section{Scoping the literature}

Systematic searches \& handsearching for articles relevant to chronic obstructive pulmonary disease, frailty \& exercise-based interventions.

Developed preliminary theory areas (considering context, mechanisms \& outcomes).

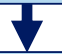

Refining the review focus with stakeholders

Preliminary theories areas explored \& shortlisted with stakeholders to refine the focus of the review.

\section{$\checkmark$}

Identifying relevant and rigorous evidence

Re-screened initial search results for those relevant to the refined review focus. Supplemented literature with targeted handsearching.

\section{Extracting and organising data}

Extracted insights about context, mechanisms, interventions \& outcomes for each shortlisted theory area, considering relevance \& rigor.

\section{Analysis and synthesis}

Summary \& synthesis around each shortlisted theory area, incorporating stakeholder input.

Figure I Methods summary for this realist review.

give their reflections on early findings to help us develop the final synthesis. Members of the review team [LB, MM] typically engaged with stakeholders through informal oneto-one or group discussions, and took discussion notes to keep an audit trail.

\section{Scoping the Literature}

We began with a scoping exercise to develop evidence-based preliminary theories: ideas about how exercise-based interventions lead to their intended outcomes (also known as mechanisms) in the context of living with both COPD and frailty. To identify key articles, we conducted structured searches of the following electronic databases: British Nursing Index; CINAHL; Cochrane Database of Systematic Reviews and Central Register of Controlled Trials; EMBASE; MEDLINE; PsycINFO; and the Web of Science Core Collection. We ran searches on January 10th 2019, and later updated the search to October 23rd 2019. We used the broad search structure of "[COPD] AND [FRAILTY] AND [EXERCISE]", using a combination of subject headings and free text searches of titles and abstracts (full search strategy available on PROSPERO). We identified further records through handsearching, including drawing on forward and backwards citation tracking, personal libraries, and recommendations from expert stakeholders.

We screened the results of the searches inclusively for articles addressing exercise-based interventions, exercise barriers and facilitators, and/or experiences or perceptions of exercise in people living with COPD and frailty. There were no restrictions by design, or year or language of publication.

We defined exercise as "planned, structured, and repetitive bodily movement with the objective of improving or maintaining at least one aspect of physical fitness" ${ }^{35}$ (e.g. pulmonary rehabilitation, walking programs, yoga). Exercise had to be a core component of the intervention described but could be part of a multicomponent intervention.

For our population, we included studies where participants had a clinical diagnosis of COPD as defined by GOLD guidelines; ${ }^{36}$ and were aged $\geq 35$ years. ${ }^{37}$ Mixed populations were included if $\geq 50 \%$ met these criteria, or if information about these participants could be separated. Where limited evidence was available, we also drew from the wider chronic respiratory disease literature. We prioritised studies where frailty was determined using a pre-defined, referenced measure (e.g. Fried Frailty Phenotype, ${ }^{38}$ Short Physical Performance Battery ${ }^{39,40}$ Clinical Frailty Scale, ${ }^{41}$ PaulsonLichtenberg Frailty Index ${ }^{42}$ ). Where evidence from studies using such measures was limited, we also drew on studies where other indicators suggested a frail population (e.g. poor physical function, limited activities of daily living, slow gait speed).

\section{Refining the Focus of the Review with Stakeholders}

We read and re-read included articles for familiarisation, and then analysed them thematically to develop a list of preliminary theories about how and in what circumstances exercise-based interventions work best for people with COPD and frailty. We discussed the preliminary theories with stakeholders to refine and derive a list of the three to five most salient for future practice and research. We then used this shortlist to refine the scope of the review and direct the subsequent stages.

\section{Identifying Relevant and Rigorous Evidence}

We then conducted revised searches for evidence relevant to the shortlisted preliminary theories. This included re-assessing the results of the scoping search and 
supplementing the results with more targeted handsearching. The aim of this supplementary searching was not to identify an exhaustive set of research studies, but to reach a point of theoretical saturation within the synthesis.

In addition to meeting the criteria above in relation to exercise-based interventions, COPD and frailty, articles needed to include insights relevant to the shortlisted preliminary theories to be included in the final synthesis. We reviewed full-text articles to determine if they contained enough detail or theoretical discussion to contribute to our aims. ${ }^{43}$ For transparency, we noted whether the relevance of contributions were high (well aligned to the review questions, with rich descriptions contributing to theory); moderate (some relevance to review question and some theoretical insights) or low (meets broad inclusion criteria and provides at least one insight).

As realist reviews may use part of an article that is relevant (e.g. subgroup analyses rather than main trial findings), standardised assessment of studies as a whole is less helpful. ${ }^{33}$ Instead, we considered the rigour of the data or inference for the purpose it is being used within the synthesis, and prioritised contributions accordingly (i.e. studies deemed methodologically weaker were prioritised less in the synthesis). Depending on the risk of bias, contributions were categorised as high, medium or low quality.

\section{Extracting and Organising Data}

We used tailored data extraction forms to support the realist synthesis. For each article, we extracted descriptive details (i.e. aims, design, etc.) and interventions used (where applicable). For each shortlisted preliminary theory, we extracted important contextual factors, intervention outcomes, and implied or actual mechanisms involved in achieving desired outcomes. Data were extracted from methods and results sections where possible. In line with previous realist reviews, we also extracted authors' discussions about important contextual factors and mechanisms to include these insights as data within the review. ${ }^{43}$

\section{Analysis and Synthesis}

We used the extracted information to develop the final evidence-based theories incorporating contextual factors, mechanisms, potential intervention strategies, and outcomes. We summarised the data using a narrative and with an accompanying figure. During this process, we attended to evidence supporting these theory areas alongside contradictory examples and unintended consequences. ${ }^{44}$ We discussed areas of uncertainty within the final synthesis with stakeholders, and where helpful we conducted additional searches to refine the synthesis further.

\section{Results \\ Study Details}

Of 344 records identified through database searching and handsearching, 31 contributed to the initial scoping phase of the review (Figure 2). Of these, 16 were included in the final synthesis, supplemented with four additional articles. All 35 articles included in the scoping and/or final synthesis are shown in Table 1. Publication dates ranged from 1996 to 2019 (over 75\% published in the past 5 years). The most common countries represented were the UK $(n=8)$, USA $(n=7)$, and the Netherlands $(n=6)$, followed by Australia $(n=3)$, Italy $(n=2)$, and one each from Belgium, China, Denmark, France, Ireland, Mexico, Poland, Spain, and Sweden. Twenty-one were primary studies and 14 were reviews/commentaries.

\section{Focus of Included Studies}

Of four randomised trials, three tested the effectiveness of exercise-based interventions (a hospital-based pedal exercise intervention, ${ }^{45}$ pulmonary rehabilitation with or without electrical muscle stimulation, ${ }^{46}$ and home-based pulmonary rehabilitation ${ }^{47}$ ); one trial tested whether the severity of respiratory disability affected outcomes of centre- or homebased pulmonary rehabilitation. ${ }^{48}$ Of 12 cohort studies, most assessed the impact of pulmonary rehabilitation on frailty or frailty components, ${ }^{49-54}$ three assessed the feasibility or outcomes of geriatric rehabilitation, ${ }^{55-57}$ and three assessed how baseline characteristics (e.g. dyspnoea severity, exercise tolerance, frailty) affect pulmonary rehabilitation outcomes. ${ }^{10,58,59}$ Four qualitative studies explored experiences of, and reasons for (non-)participation in, exercise-based interventions (primarily pulmonary rehabilitation ${ }^{60-63}$ ). One case series described three peoples' journeys through geriatric rehabilitation. ${ }^{64}$

Most of the reviews examined the interplay between relevant geriatric syndromes (e.g. frailty, sarcopenia, multimorbidity) and respiratory disease, ${ }^{1,19,65,66}$ plus appropriate interventions in this context. ${ }^{67-69}$ Other reviews focused on frailty assessment ${ }^{70}$ and optimising physical activity and pulmonary rehabilitation interventions. ${ }^{21,71,72}$ We also included a letter about pulmonary rehabilitation in severe $\mathrm{COPD},{ }^{73}$ an editorial on frailty, comorbidity and COPD, ${ }^{74}$ and a commentary on a geriatric policy document from a lung health task force. ${ }^{75}$ 


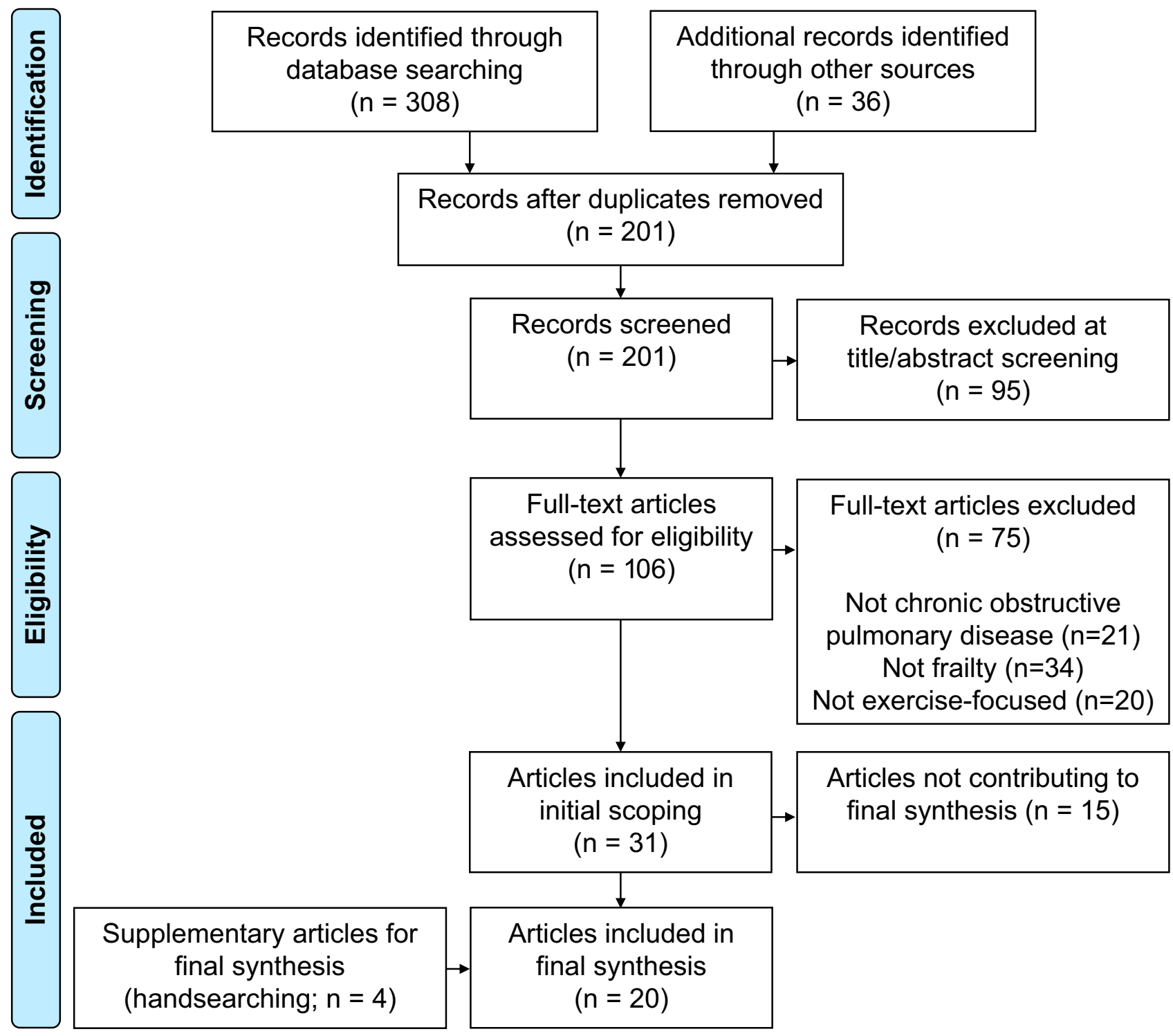

Figure 2 Flow of chart of articles included in the review.

\section{Scoping the Literature}

The Context of Living with Frailty

Authors noted how this population frequently present with lower levels of function and health, and higher levels of dyspnoea, distress relating to dyspnoea, disability, and comorbidities, ${ }^{10,19,21,48,59-68,73,74}$ including comorbid psychiatric illness and cognitive dysfunction. ${ }^{56,61}$ This was further complicated by their risk of significant health events or deterioration during exercise-based interventions. ${ }^{10,19,21,61,64,68}$ People's health influenced how they saw themselves and services, contributing to feelings of shame ${ }^{60}$ or losses of control. $^{62,63,66}$ Participants reported feeling too unwell or too frail to participate in exercise, ${ }^{60,61,63,65}$ especially in the context of other comorbidities. ${ }^{60,63}$ Our service user and professional stakeholders also felt social isolation was important, acting as both a barrier to, and facilitator of, participation. Altogether, these factors influenced individuals' perceptions of exercise-based interventions in relation to their priorities, ${ }^{60-63,69}$ as well as their physical ability to engage.

\section{Factors Affecting Participation and Outcomes}

Our preliminary theories identified several factors affecting participation in, and outcomes of, exercise-based interventions in this context. These factors are illustrated in Figure 3.

Authors recommended actively involving people within exercise-based interventions to help them feel more worthy and in control. ${ }^{60,62,64,69,75}$ Incorporating better communication and relationships with staff ${ }^{47,60-62}$ was 
Table I Studies Included in the Review

\begin{tabular}{|c|c|c|c|c|c|c|c|}
\hline $\begin{array}{l}\text { Contribution to } \\
\text { Synthesis }\end{array}$ & Author & Year & Design & Diagnoses & Frailty & $\begin{array}{l}\text { Exercise-Based } \\
\text { Intervention }\end{array}$ & $\begin{array}{l}\text { Contribution } \\
\text { Rigour }\end{array}$ \\
\hline High & $\begin{array}{l}\text { Boxall et al }{ }^{47} \\
\text { Brighton et } \mathrm{al}^{61} \\
\text { Harrison et } \mathrm{al}^{62}\end{array}$ & $\begin{array}{l}2005 \\
\text { (unpublished) } \\
2015\end{array}$ & $\begin{array}{l}\text { RCT } \\
\text { Qualitative } \\
\text { Qualitative }\end{array}$ & $\begin{array}{l}\text { COPD } \\
\text { COPD } \\
\text { COPD }\end{array}$ & $\begin{array}{l}\text { Inferred } \\
\text { Specified } \\
\text { Inferred }\end{array}$ & $\begin{array}{l}\text { Home-based PR } \\
\text { PR } \\
\text { PR }\end{array}$ & $\begin{array}{l}\text { Medium } \\
\text { High } \\
\text { High }\end{array}$ \\
\hline Medium & $\begin{array}{l}\text { Benzo et al } \\
\text { Crisafulli et al }^{60} \\
\text { Holland et al } \\
\text { Thorpe et al }{ }^{69} \\
\text { Van Dam van Isselt } \\
\text { et al } \\
\text { Van Dam van Isselt } \\
\text { et al }{ }^{64}\end{array}$ & $\begin{array}{l}2015 \\
2014 \\
2016 \\
2012 \\
2019 \\
2013\end{array}$ & $\begin{array}{l}\text { Qualitative } \\
\text { Literature } \\
\text { review } \\
\text { Literature } \\
\text { review } \\
\text { Systematic } \\
\text { review } \\
\text { Cohort study } \\
\text { Case series }\end{array}$ & $\begin{array}{l}\text { COPD } \\
\text { COPD } \\
\text { COPD } \\
\text { COPD } \\
\text { COPD } \\
\text { COPD }\end{array}$ & $\begin{array}{l}\text { Inferred } \\
\text { Inferred } \\
\text { Inferred } \\
\text { Inferred } \\
\text { Inferred } \\
\text { Inferred }\end{array}$ & $\begin{array}{l}\text { PR } \\
\text { PR } \\
\text { PR } \\
\text { Physical activity } \\
\text { Geriatric rehabilitation } \\
\text { Geriatric rehabilitation }\end{array}$ & $\begin{array}{l}\text { High } \\
\text { Low } \\
\text { Low } \\
\text { Medium } \\
\text { Medium } \\
\text { Low }\end{array}$ \\
\hline Low & 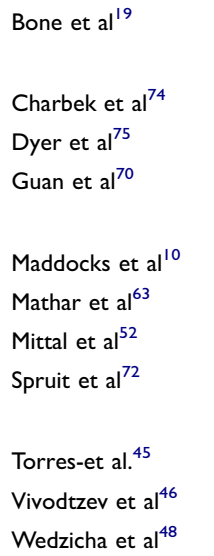 & $\begin{array}{l}2017 \\
2018 \\
2019 \\
2018 \\
\\
2018 \\
2017 \\
2015 \\
2019 \\
\\
2017 \\
2006 \\
1998\end{array}$ & $\begin{array}{l}\text { Literature } \\
\text { review } \\
\text { Editorial } \\
\text { Commentary } \\
\text { Literature } \\
\text { review } \\
\text { Cohort study } \\
\text { Qualitative } \\
\text { Cohort study } \\
\text { Literature } \\
\text { review } \\
\text { RCT } \\
\text { RCT } \\
\text { RCT }\end{array}$ & $\begin{array}{l}\text { Lung disease } \\
\text { COPD } \\
\text { Lung disease } \\
\text { COPD } \\
\text { COPD } \\
\text { COPD } \\
\text { Lung disease } \\
\text { COPD } \\
\text { COPD } \\
\text { COPD } \\
\text { COPD }\end{array}$ & $\begin{array}{l}\text { Specified } \\
\text { Specified } \\
\text { Inferred } \\
\text { Specified } \\
\text { Specified } \\
\text { Inferred } \\
\text { Specified } \\
\text { Inferred } \\
\text { Specified } \\
\text { Inferred } \\
\text { Inferred }\end{array}$ & $\begin{array}{l}\text { PR } \\
\text { Exercise (general) } \\
\text { PR } \\
\text { PR } \\
\text { PR } \\
\text { PR } \\
\text { PR } \\
\text { PR } \\
\text { Hospital-based exercise } \\
\text { PR plus ES } \\
\text { PR/Home-based PR }\end{array}$ & $\begin{array}{l}\text { Low } \\
\text { Low } \\
\text { Low } \\
\text { Low } \\
\text { Medium } \\
\text { Medium } \\
\text { Low } \\
\text { Medium } \\
\text { Medium } \\
\text { Medium } \\
\text { High }\end{array}$ \\
\hline Scoping only & 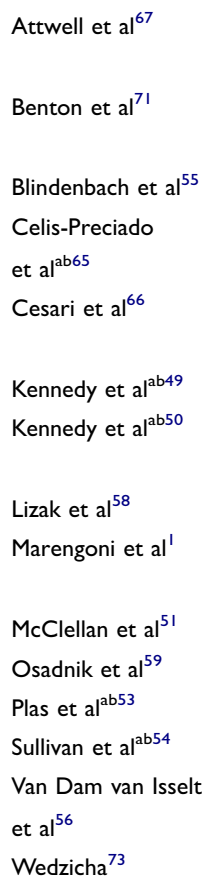 & $\begin{array}{l}2017 \\
2006 \\
2017 \\
2017 \\
2014 \\
2015 \\
2018 \\
2008 \\
2018 \\
2014 \\
2018 \\
2019 \\
2016 \\
2014 \\
1996\end{array}$ & $\begin{array}{l}\text { Literature } \\
\text { review } \\
\text { Literature } \\
\text { review } \\
\text { Cohort study } \\
\text { Systematic } \\
\text { review } \\
\text { Literature } \\
\text { review } \\
\text { Cohort study } \\
\text { Cohort study } \\
\text { Cohort study } \\
\text { Systematic } \\
\text { review } \\
\text { Cohort study } \\
\text { Cohort study } \\
\text { Cohort study } \\
\text { Cohort study } \\
\text { Cohort study } \\
\text { Letter }\end{array}$ & $\begin{array}{l}\text { Lung disease } \\
\text { COPD } \\
\text { COPD } \\
\text { COPD } \\
\text { COPD } \\
\text { Lung disease } \\
\text { Lung } \\
\text { transplant } \\
\text { COPD } \\
\text { COPD } \\
\text { Lung disease } \\
\text { COPD } \\
\text { COPD } \\
\text { COPD } \\
\text { COPD } \\
\text { COPD }\end{array}$ & $\begin{array}{l}\text { Specified } \\
\text { Inferred } \\
\text { Specified } \\
\text { Specified } \\
\text { Inferred } \\
\text { Specified } \\
\text { Specified } \\
\text { Inferred } \\
\text { Specified } \\
\text { Inferred } \\
\text { Inferred } \\
\text { Specified } \\
\text { Specified } \\
\text { Inferred } \\
\text { Inferred }\end{array}$ & $\begin{array}{l}\text { PR } \\
\text { PR } \\
\text { Geriatric rehabilitation } \\
\text { PR } \\
\text { Exercise (general) } \\
\text { PR } \\
\text { PR } \\
\text { PR } \\
\text { PR } \\
\text { PR } \\
\text { PR } \\
\text { PR } \\
\text { PR } \\
\text { Geriatric rehabilitation } \\
\text { PR/Home-based PR }\end{array}$ & $\begin{array}{l}\text { NA } \\
\text { NA } \\
\text { NA } \\
\text { NA } \\
\text { NA } \\
\text { NA } \\
\text { NA } \\
\text { NA } \\
\text { NA } \\
\text { NA } \\
\text { NA } \\
\text { NA } \\
\text { NA } \\
\text { NA } \\
\text { NA }\end{array}$ \\
\hline
\end{tabular}

Note: ${ }^{\text {ab }}$ abstract only.

Abbreviations: RCT, Randomised controlled trial; COPD, Chronic Obstructive Pulmonary Disease; PR, Pulmonary Rehabilitation; ES, Electrical stimulation; NA, Not Applicable. 
also suggested to influence how people view services in relation to themselves. ${ }^{60,61,63,65}$ Service users acknowledged the importance of relationships with intervention staff as a facilitator of attendance. Professional stakeholders agreed that it could be important to challenge preconceptions around the inevitability of frailty, and negative views of exercise. Once people felt supported to engage with exercise-based interventions, articles suggested experiencing subsequent improvements could also motivate participants going forwards. ${ }^{58,65}$

Authors also signalled the need to take a more wholeperson approach, 1,19,48,55,56,60,61,63,64,68-70,74 including having appropriate staffing and resources to do so. $55,56,64,69$ Both service user and professional stakeholders perceived a holistic approach as essential. Service users felt a broader approach helped respond to individual differences. Professionals emphasised that this was needed to identify and address the multiple concurrent issues this population experience (e.g. comorbidities, cognitive decline, poor nutritional status, socioeconomic barriers). However, this was not always possible within current exercise-based interventions like pulmonary rehabilitation. Professionals felt their practice was holistic within the realm of respiratory care, but this could be improved by taking an approach that considers each person's priorities and concerns including and beyond living with COPD.

On a practical level, authors referred to the importance of service configurations in being perceived as acceptable. This included choosing appropriate locations and timing to support engagement with exercise-based interventions, ${ }^{19,45,47,48,60,63,75}$ and appropriate tailoring of services for individuals. $1,46,48,50,55,60,62,64,66,69,70,73$ Stakeholders acknowledged that people with COPD and frailty may benefit from a modified or more flexible service structure. However, this was less often considered in existing practice.

To maximise outcomes of exercise-based interventions, authors suggested being inclusive of people with lower baseline scores and high potential for improvements. ${ }^{51,58,66}$ However, an inclusive stance needed to be balanced with evidence that low exercise tolerance or severe breathlessness can hinder benefit in some circumstances. ${ }^{48,59}$ Professional stakeholders acknowledged tensions between lower baseline abilities and judging potential to benefit from services like outpatient pulmonary rehabilitation, especially when completion rates are used as a measure of service "quality". Authors also suggested tailoring education and exercises to participants' abilities ${ }^{47,66,69}$ and ensuring people were engaging in sufficient exercise to have a measurable impact. ${ }^{48,71}$ Service users and professionals felt that within-service tailoring of exercise to abilities and restrictions was generally done well.

\section{Five core principles for exercise-based interventions for people with COPD and frailty}

Based on the scoping results and discussions with stakeholders, we identified key elements of the context of living with frailty, and mechanisms which may help this group to

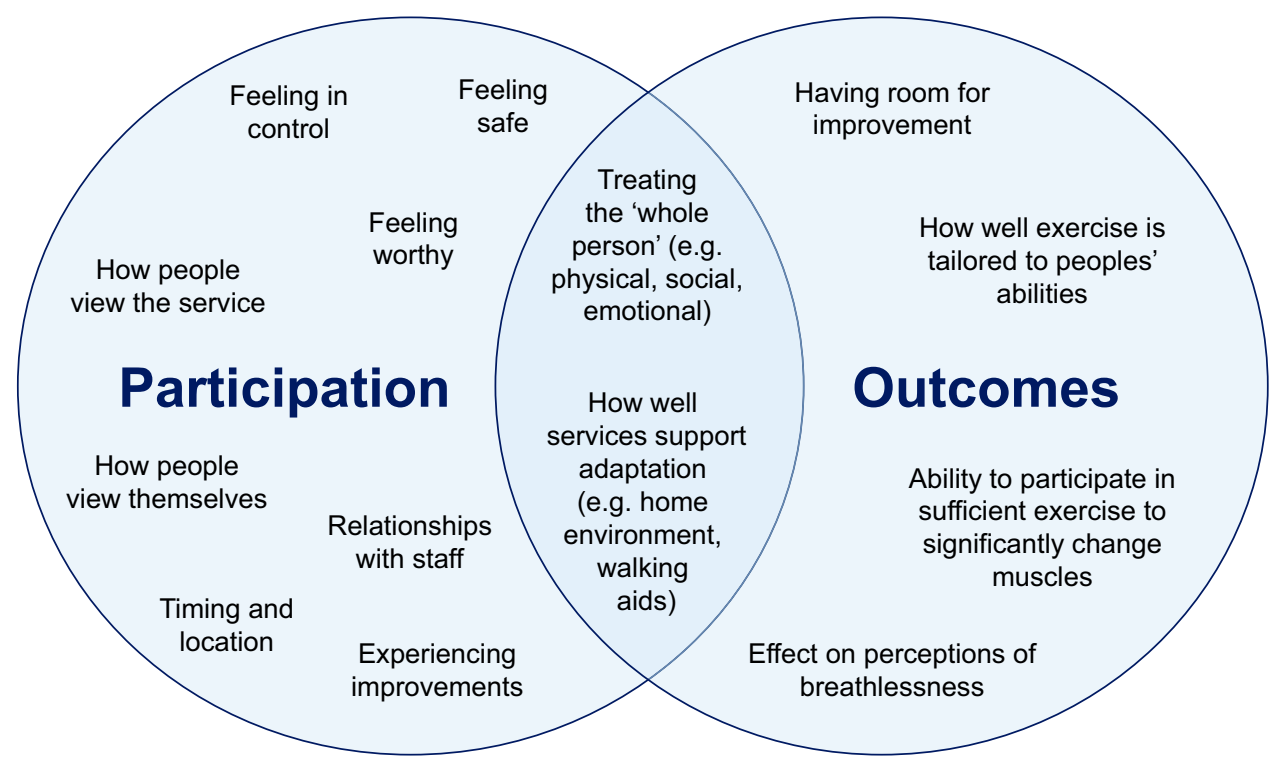

Figure 3 Factors affecting participation in, and outcomes of, exercise-based interventions for people with chronic obstructive pulmonary disease and frailty. 
benefit as much as possible from exercise-based interventions (Figure 4).

\section{Building Trusting Relationships}

Considering the context of prevalent negative self- and/or service perceptions, efforts to build trusting relationships may help to foster engagement in exercise-based interventions. Two studies suggested the importance of a therapeutic alliance between service users and those introducing and delivering exercise. ${ }^{61,62}$ Harrison et al ${ }^{62}$ suggest empathetic interactions with healthcare professionals are important to increase participants' sense of selfworth and subsequent engagement, especially in instances of low self-worth, perceived loss of control, and avoidance of professional input. Participants in Brighton et al ${ }^{61}$ noted how a safe and encouraging atmosphere supported by skilled and encouraging staff facilitated pulmonary rehabilitation attendance. This relationship-building was important in addressing their fears around exercising "at their age" or with particular comorbidities. These relationships also meant service-users felt they could reach out when sessions needed to be cancelled or moved due to an unpredictable disruption. In practice, both studies suggest empathetic communication, reassurance, and collaborative approaches to help people feel trust in their care provider and feel involved in their treatment. By overcoming fears and perceived barriers, participants can be supported to see how an exercise-based intervention might align with their priorities. ${ }^{61,62}$

The importance of building trusting relationships is supported by the success of a home-based exercise intervention by Boxall et al. ${ }^{47}$ The authors reflect how a home environment and tailored education may have helped lower anxiety and build rapport, supporting the good adherence seen in their study. Having frequent contact with staff who adjust and monitor an intervention; a characteristic also seen in the successful geriatric rehabilitation services ${ }^{57,64}$ may also contribute. Moreover, when low uptake of exercise-based interventions was experienced by Benzo et $\mathrm{al}^{60}$ they felt that building a stronger therapeutic alliance through acknowledgement of psychological comorbidities and collaborative discussions may be required to improve uptake. In relation to pulmonary rehabilitation, two reviews ${ }^{21,69}$ agreed that trust in professionals, person-centredness and shared decision-making were important in people feeling supported, listened to, well informed, and supported to engage in exercise-based interventions.

\section{Shared Understanding of Priorities}

Another key characteristic of this population is their heterogenous presentation and often multiple comorbidities Several authors referred to the importance of

\section{FRAILTY CONTEXT}

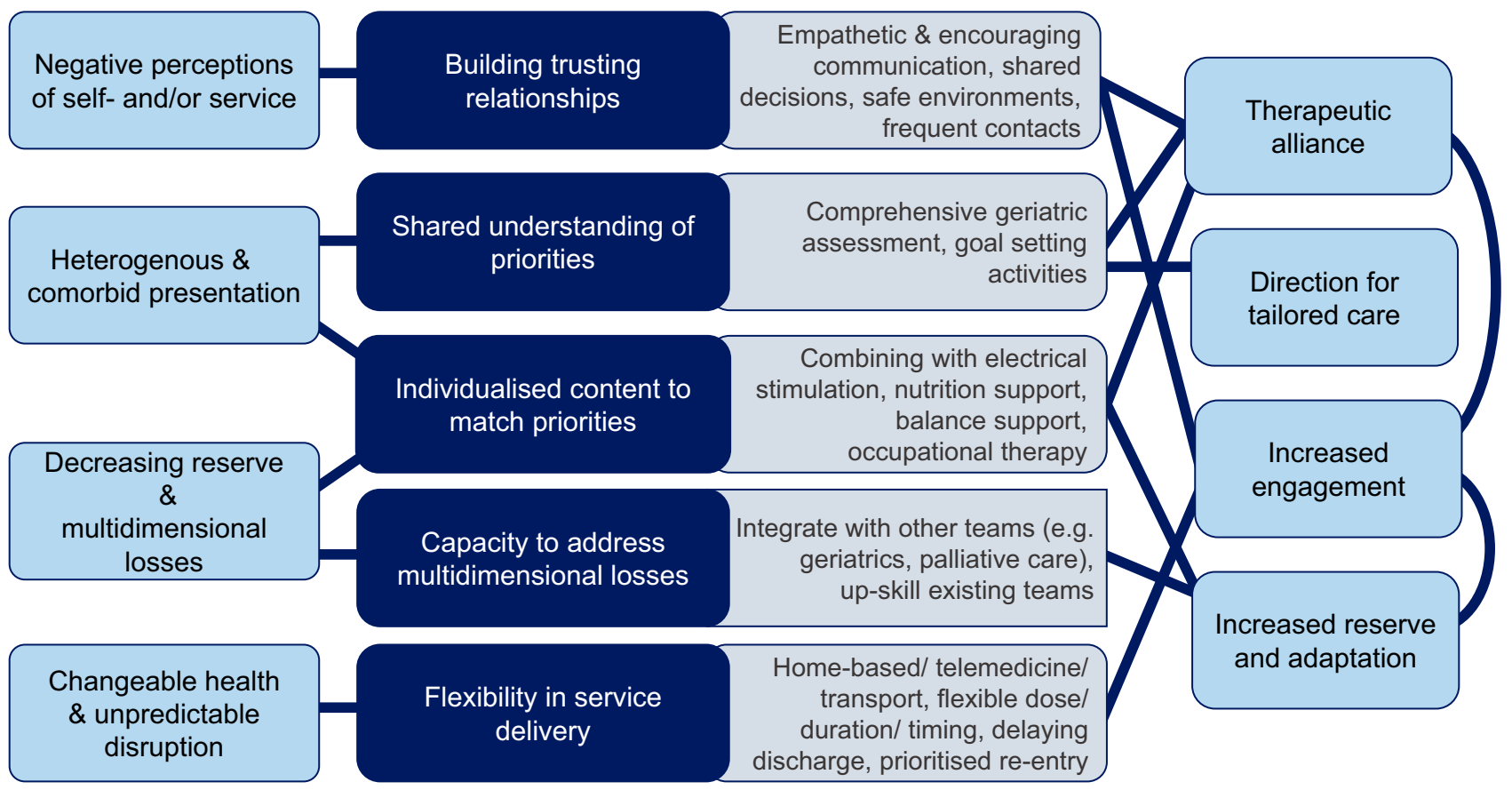

Figure 4 Core principles when adapting exercise-based interventions for people with chronic obstructive pulmonary disease and frailty. 
understanding multidimensional concerns to inform individualised approaches. ${ }^{60-62}$ Others also suggested that having a better shared understanding of priorities might support relationship-building and greater engagement. ${ }^{62} \mathrm{In}$ one study, over a third of people declined post-hospital physical activity interventions due to a lack of interest. ${ }^{60}$ The authors felt that working to understand patients' specific needs and how they might be addressed (including through physical activity) might be the key to increasing engagement in the future. Creating a shared understanding of needs and goals therefore seems essential to provide direction for tailored care.

In practice, authors commonly referred to a role for Comprehensive Geriatric Assessment, ${ }^{61,64,70,75}$ and/or goalsetting activities ${ }^{21,62,69}$ to achieve this. For example, Van Dam van Isselt et $\mathrm{al}^{64}$ report case studies from an inpatient geriatric rehabilitation service. This service begins with a Comprehensive Geriatric Assessment, in order to tailor the subsequent programme. The authors attribute perceived longer-term impacts of their service to focusing on what is most important for that individual. Meanwhile, goal-setting activities were highlighted in a review of barriers and enablers to physical activity programmes. ${ }^{21}$ Goal-setting was perceived as a motivational activity in itself, as well as being helpful in guiding the right professional support. In both instances, creating a shared understanding of participants' priorities was felt to improve the experience and impact of exercise-based interventions.

\section{Individualised Content to Match Priorities}

Having created a shared understanding of priorities, it becomes important to consider how service content can be appropriately tailored: not only at the start but also potentially adjusting throughout. Individualising content may allow interventions to better align with the diverse and fluctuating concerns seen within this population.

Following a comprehensive assessment within geriatric rehabilitation, van Dam van Isselt et $\mathrm{al}^{64}$ give examples of how their intervention can be individualised. For example, in one case, they prioritised more psychological approaches for someone whose main concerns were around accepting a loss of independence. For another, they prioritised advanced care planning activities due to the advanced nature of his disease. They hypothesise that by focusing on a person's specific problems there is scope for greater impact. Indeed, their subsequent cohort study suggests positive effects on quality of life using this approach. ${ }^{57}$ Similarly, Vivodtzev et al ${ }^{46}$ show how applying a specific approach (e.g. electrical muscle stimulation) to a group most likely to benefit (in this instance, people with low body mass index who had a recent exacerbation of their COPD) can result in improved outcomes (increased muscle strength, reduced dyspnoea during activity). Two reviews ${ }^{21,69}$ also highlight evidence that people with COPD prefer individualised models of care that target their priorities, and suggest this as an essential component of person-centred approaches. This is in line with Harrison et al's ${ }^{62}$ suggestion that individualised approaches may make pulmonary rehabilitation more meaningful and encourage more active participation, as well as supporting therapeutic alliance between service-users and practitioners. Practical suggestions have included identifying where specific individuals may benefit from combining exercise-based approaches with other interventions, including: non-invasive ventilation or oxygen, ${ }^{64,72}$ neuromuscular electrical stimulation or whole-body vibration, ${ }^{46,72}$ occupational therapy, ${ }^{64,68}$ specific postural or balance interventions, ${ }^{68}$ nutritional support, ${ }^{64,68,70}$ geriatric input ${ }^{61,64}$ and/or palliative care. ${ }^{63,64}$

However, the influence of individualisation on engagement is mixed. A high rate of decline was reported for the inpatient geriatric rehabilitation service ${ }^{57}$ (approximately $50 \%)$ despite the content being tailored around specific needs. ${ }^{64}$ Yet, a home-based service that took a tailored approach (e.g. adjusting exercises around pain and individualising the education delivered) reported a higher than usual participation rate, ${ }^{47}$ which they attributed to individuation as well as the removal of travel barriers. In interviews, participants do describe appropriate tailoring of exercises within pulmonary rehabilitation (e.g. working around historic injuries) as part of what contributes to a safe and encouraging atmosphere. ${ }^{61}$ It may be that within-service tailoring is important in achieving positive experiences and outcomes as input is carefully matched to need, but that more structural flexibility of services (e.g. moving to a home-based approach ${ }^{47}$ ) is more important for initial engagement.

\section{Capacity to Address Multidimensional Losses}

To provide individualised approaches, exercise-based interventions must also have the capacity to address losses of reserve and concerns that are multidimensional (i.e. including physical, psychological and social aspects), including non-respiratory comorbidities.

Multidimensional approaches are thought to support both engagement and outcomes of exercise-based interventions, but the evidence around engagement again is mixed. Benzo $\mathrm{t} \mathrm{al}^{60}$ offered people hospitalised for their COPD 
either pulmonary rehabilitation, or a less burdensome activity monitoring approach. Most participants (75\%) declined both, including a large proportion (39\%) citing a lack of interest as the main reason. As a result, they proposed that better matching services to patients' needs and interests may be more effective in improving engagement than simply reducing burden. Yet, high rates of decline are still seen for multidisciplinary approaches: approximately $50 \%$ of those offered van Dam van Isselt et al's ${ }^{57}$ geriatric rehabilitation service declined, and approximately $45 \%$ of those with frailty did not complete the multidisciplinary pulmonary rehabilitation in Maddocks et al's study. ${ }^{10}$ As with individualising content, offering a multidisciplinary approach does not seem enough on its own to promote greater engagement.

The more evident benefit of multidisciplinary approaches seemed to be in the capacity to impact on broader patient groups and health outcomes. For example, a less multidisciplinary pulmonary rehabilitation programme was fairly effective for non-housebound COPD patients but was not sufficient to improve outcomes in those who were housebound. $^{48}$ Moreover, a hospital-based intervention focused solely on exercise was only effective in improving physical outcomes (e.g. muscle strength, balance), ${ }^{45}$ and a respiratory-focused and -led programme was successful in improving gait speed but not overall frailty. ${ }^{52}$

Meanwhile, people who attended a pulmonary rehabilitation programme with education delivered by a multidisciplinary team showed improved health status, physical and psychological outcomes compared to baseline. ${ }^{10}$ People who received inpatient geriatric rehabilitation (incorporating input from physicians, nurses, physiotherapists, occupational therapists, and psychologists, with integration across respiratory, geriatrics and palliative care), although showing no difference in walking distance and daily activities, showed greater improvements on disease-specific quality of life and reduced exacerbations compared to people receiving a solely physiotherapy-based approach. ${ }^{57}$ In addition, people with COPD who were housebound receiving a home-based multidisciplinary pulmonary rehabilitation programme (including physiotherapy, nursing, and occupational therapy input) showed improved exercise tolerance, perception of breathlessness and quality of life compared to a wait-list control group. ${ }^{47}$

As a result, there is a consistent narrative around the importance of multidisciplinary approaches for this population. ${ }^{69,70}$ Practical suggestions include incorporating input around, for example, nutrition, polypharmacy, and occupational therapy input, ${ }^{19,61,68,74}$ integration between teams (e.g. respiratory, geriatrics, palliative care) and/or ensuring more "holistic" pulmonary rehabilitation delivery. ${ }^{19,68,69,72}$ However, scepticism remains around how well these strategies are implemented in practice.

\section{Flexibility in Service Delivery}

Changeable health and risk of unpredictable disruptions are commonly experienced by this population, and engagement in more individualised and multidisciplinary exercise-based interventions can still be limited. Services may, therefore, need to consider more flexibility in delivery to increase engagement.

The most commonly proposed service adaptation for this and similar populations is offering a home-based alternative to centre-based pulmonary rehabilitation. ${ }^{4,60,63,68}$ However, while Wedzicha et $\mathrm{al}^{48}$ showed that both centre- and homebased strategies can receive similar levels of engagement from participants, offering a home-based physical activity alternative does not always avoid high numbers of people declining exercise-based interventions. ${ }^{60}$ It has been argued that simply offering home-based pulmonary rehabilitation may, in fact, ignore the complexity and heterogeneity of some people with COPD, and may further fragment the required interdisciplinary care the most complex patients require. ${ }^{72}$ Other dimensions of flexibility may therefore be helpful to consider.

For example, two studies reported the ability to increase the "dose" of their intervention where required, by providing additional visits within their home-based pulmonary rehabilitation $^{47}$ or extending the duration of geriatric rehabilitation. ${ }^{64}$ Adjusting the service dose appeared particularly beneficial in the former, where they noted higher participation than similar studies, and that experiencing an exacerbation during the study did not prevent people from completing the intervention. The geriatric rehabilitation service also experienced good engagement with their inpatient programme once people agreed to take part, ${ }^{57}$ but it is possible that the less flexible aspects of their service (e.g. requiring a 6-week inpatient stay) were less appealing to the $50 \%$ of people who declined to participate. Alternatives suggested by others include flexible class scheduling, ${ }^{19}$ transport schemes, ${ }^{19}$ incorporating telemedicine, ${ }^{63}$ making additional contacts before discharge when people do not attend, and/or prioritising peoples' re-entry into services when unpredictable disruptions to their health occur. ${ }^{61}$

\section{Discussion}

This review highlights several important contextual factors when planning exercise-based interventions for people with both COPD and frailty. These include service-users' 
negative beliefs about themselves and exercise services, heterogenous presentation and comorbidities, decreased reserves and multidimensional loss, and experiences of unpredictable health and disruptions. Mechanisms that offer most opportunities to improve outcomes in these circumstances include approaches that try to build trusting relationships and create a shared understanding of needs, individualise approaches to priorities, have the capacity to address multidimensional concerns, and offer flexible delivery options. These mechanisms are particularly critical in the context of frailty, focusing on exercise-based interventions due to their effectiveness in improving frailty status and wider health outcomes when this population is able to engage. ${ }^{10}$ They are also likely to be important for many people with COPD not living with frailty, and for aspects of care beyond exercise-based interventions.

Our findings resonate with ideas around needing more tailored interventions for people with frailty ${ }^{76}$ and respiratory disease, ${ }^{77}$ thinking creatively about engagement with exercise-based interventions, ${ }^{78}$ and taking integrated approaches to support the maintenance of physical and mental capacities in an aging population. ${ }^{79}$ Recent examples within wider respiratory care include interdisciplinary models of primary care for people with $\mathrm{COPD},{ }^{80}$ integrated respiratory and palliative care services for chronic breathlessness, ${ }^{81}$ and rehabilitation for people with both respiratory and heart disease. ${ }^{82}$ As research specifically with people with COPD and frailty increases, ${ }^{50,54}$ this type of complex interventional work will be a priority. However, alongside this, we must also address the current lack of explicit theorising around intervention and outcome selection, and an absence of mixed methods work to explore these ideas empirically. These techniques contribute to learning beyond specific interventions, settings, and populations, and inform how learning can be transferred to different contexts. ${ }^{29,83}$

Our five evidence-driven priorities for exercise-based interventions for people with COPD and frailty (Box 1) may be useful to those working in service development

Box I Five Priorities for Exercise-Based Interventions for People with Both Chronic Obstructive Pulmonary Disease and Frailty

\begin{tabular}{|c|l|}
\hline I. & Build trusting relationships \\
2. & Develop a shared understanding of the service user's priorities \\
3. & Individualise content to match the service user's priorities \\
4. & Ensure capacity to address multidimensional losses \\
5. & Offer flexibility in service delivery \\
\hline
\end{tabular}

and delivery to identify areas for improvement. Both local stakeholder feedback and wider international examples of services included in this review suggest that current practice is varied in relation to these priorities. For example, an international survey of pulmonary rehabilitation programs found that while many services include multi-dimensional interventions and multi-professional teams, few reported actively using goal setting with service-users. ${ }^{72}$ Moreover, many services are experiencing increased referrals, reduced staff-patient ratios, and pressures to reduce waiting times. ${ }^{84}$ This context likely makes it challenging for teams to invest their time and resources in building trusting relationships, individualising content and considering flexible approaches when needed.

We have indicated practical suggestions (shown in Figure 4) that might support effective exercise-based approaches for people with COPD and frailty. For example, building capacity to address multidimensional losses might involve upskilling existing team members in identifying and addressing common components of frailty, or creating integrated pathways with geriatric specialists. Such interventions can be selected to optimise efficiency by tackling multiple priorities with one approach. For example, home-based approaches may contribute to flexibility and support engagement for some, ${ }^{85}$ but may offer less scope for tailoring input (beyond exercise) and fail to address multidimensional losses. However, integrating a Comprehensive Geriatric Assessment in collaboration with geriatric specialists might contribute to a shared understanding of needs, relationshipbuilding, and support capacity to both individualise approaches and address multidimensional losses. Such approaches can be used to build on (rather than replace) established approaches like pulmonary rehabilitation. Importantly, any new approaches should be developed in collaboration with service users, tested through mixedmethods approaches, and reported with explicit consideration of intervention theory.

Taking a realist approach to this review allowed us to synthesise a diverse range of evidence and insights in order to answer questions around how and why some approaches may be particularly beneficial for this population. We incorporated professionals' and service-users' views alongside published literature to refine the review focus to areas deemed most pertinent to future practice. Moreover, this style of synthesis shifts the focus from specific interventions and services to broader underlying mechanisms or principles. This approach better acknowledges the reality that in practice, strategies will need to 
vary according to existing service configurations. In our protocol, we planned to conduct further database searches after the scoping exercise. However, the limited literature available in this first step suggested targeted handsearching would be a more efficient approach to supplement the synthesis. As our synthesis was limited by infrequent explicit consideration of how and why exercise-based their interventions were effective (or not), the potentially important mechanisms we outline should be treated as hypotheses that will require further testing and refinement.

\section{Conclusions}

In order to increase engagement with, and outcomes from, exercise-based interventions for people with both COPD and frailty, we may need to build trusting relationships; create a shared understanding of needs; individualise approaches to their priorities, ensure capacity to address multidimensional concerns; and use flexible approaches to intervention delivery. Future work to develop and test different strategies to implement these principles across different contexts will be essential in optimising outcomes for this group.

\section{Acknowledgments}

Thank you to all the patient, informal carer, public representatives who contributed to the study design and interpretation of the findings, including members of the BRC Respiratory PPI group, the Cicely Saunders Institute public involvement group, and project team members from the Harefield Breathing Group. Thank you to the professional stakeholders who contributed to discussions in refining the scope of the review, and to Patricia Wilson who provided feedback on the initial review protocol. Thank you also to Simon Etkind for providing constructive feedback on the structure and clarity of the manuscript.

\section{Funding}

This project is funded by a National Institute for Health Research (NIHR) Career Development Fellowship (CDF2017-10-009) held by MM. This research was supported by the NIHR Collaboration for Leadership in Applied Health Research and Care South London, now recommissioned as NIHR Applied Research Collaboration South London. CE is funded by a Health Education England/ NIHR Senior Clinical Lectureship. This publication presents independent research funded by the National Institute for Health Research (NIHR). The views expressed in this publication are those of the author(s) and not necessarily those of the NHS, NIHR or the Department of Health and Social Care.

\section{Disclosure}

Dr Matthew Maddocks reports grants from NIHR, outside the submitted work. The authors declare that there is no conflict of interest in this work.

\section{References}

1. Marengoni A, Vetrano DL, Manes-Gravina E, Bernabei R, Onder G, Palmer K. The relationship between COPD and frailty: a systematic review and meta-analysis of observational studies. Chest. 2018;154 (1):21-40. doi:10.1016/j.chest.2018.02.014

2. Rodriguez-Manas L, Feart C, Mann G, et al. Searching for an operational definition of frailty: a Delphi method based consensus statement: the frailty operative definition-consensus conference project. $J$ Gerontol A Biol Sci Med Sci. 2013;68(1):62-67. doi:10.1093/gerona/gls119

3. Morley JE, Vellas B, van Kan GA, et al. Frailty consensus: a call to action. J Am Med Dir Assoc. 2013;14(6):392-397. doi:10.1016/j. jamda.2013.03.022

4. Jones SE, Maddocks M, Kon SS, et al. Sarcopenia in COPD: prevalence, clinical correlates and response to pulmonary rehabilitation. Thorax. 2015;70(3):213-218. doi:10.1136/thoraxjnl-2014-206440

5. Galizia G, Cacciatore F, Testa G, et al. Role of clinical frailty on long-term mortality of elderly subjects with and without chronic obstructive pulmonary disease. Aging Clin Exp Res. 2011;23 (2):118-125. doi:10.1007/BF03351076

6. Vaz Fragoso CA, Enright PL, McAvay G, Van Ness PH, Gill TM. Frailty and respiratory impairment in older persons. Am J Med. 2012;125(1):79-86. doi:10.1016/j.amjmed.2011.06.024

7. Bernabeu-Mora R, Garcia-Guillamon G, Valera-Novella E, GimenezGimenez LM, Escolar-Reina P, Medina-Mirapeix F. Frailty is a predictive factor of readmission within 90 days of hospitalization for acute exacerbations of chronic obstructive pulmonary disease: a longitudinal study. Ther Adv Respir Dis. 2017;11(10):383-392. doi: $10.1177 / 1753465817726314$

8. Kon SS, Jones SE, Schofield SJ, et al. Gait speed and readmission following hospitalisation for acute exacerbations of COPD: a prospective study. Thorax. 2015;70(12):1131-1137. doi:10.1136/ thoraxjnl-2015-207046

9. Kusunose M, Oga T, Nakamura S, Hasegawa Y, Nishimura K. Frailty and patient-reported outcomes in subjects with chronic obstructive pulmonary disease: are they independent entities? BMJ Open Respir Res. 2017;4(1):e000196. doi:10.1136/bmjresp-2017-000196

10. Maddocks M, Kon SSC, Canavan JL, et al. Physical frailty and pulmonary rehabilitation in COPD: a prospective cohort study. Thorax. 2016;71(11):988-995. doi:10.1136/thoraxjnl-2016-208460

11. Valenza MC, Torres-Sanchez I, Cabrera-Martos I, Rodriguez-Torres J, Gonzalez-Jimenez E, Munoz-Casaubon T. Physical activity as a predictor of absence of frailty in subjects with stable COPD and COPD exacerbation. Respir Care. 2016;61(2):212-219. doi:10.4187/ respcare. 04118

12. Singer JP, Lederer DJ, Baldwin MR. Frailty in pulmonary and critical care medicine. Ann Am Thorac Soc. 2016;13(8):1394-1404. doi:10.1513/AnnalsATS.201512-833FR

13. McCarthy B, Casey D, Devane D, Murphy K, Murphy E, Lacasse Y. Pulmonary rehabilitation for chronic obstructive pulmonary disease. Cochrane Database Syst Rev. 2015;2.

14. Puhan MA, Gimeno-Santos E, Scharplatz M, Troosters T, Walters EH, Steurer J. Pulmonary rehabilitation following exacerbations of chronic obstructive pulmonary disease. Cochrane Database Syst Rev. 2016;10. doi:10.1002/14651858.CD005305.pub4 
15. McNamara RJ, Spencer L, Dale M, Leung RWM, McKeough ZJ. Alternative exercise and breathing interventions in chronic obstructive pulmonary disease: a critical review. Eur Med J Res. 2018;6(1):117-127.

16. Lopez P, Pinto RS, Radaelli R, et al. Benefits of resistance training in physically frail elderly: a systematic review. Aging Clin Exp Res. 2018;30(8):889-899. doi:10.1007/s40520-017-0863-Z

17. Tulloch A, Bombell H, Dean C, Tiedemann A. Yoga-based exercise improves health-related quality of life and mental well-being in older people: a systematic review of randomised controlled trials. Age Ageing. 2018;47(4):537-544. doi:10.1093/ageing/afy044

18. Clegg AP, Barber SE, Young JB, Forster A, Iliffe SJ. Do home-based exercise interventions improve outcomes for frail older people? Findings from a systematic review. Rev Clin Gerontol. 2012;22 (1):68-78. doi:10.1017/S0959259811000165

19. Bone AE, Hepgul N, Kon S, Maddocks M. Sarcopenia and frailty in chronic respiratory disease: lessons from gerontology. Chron Resp Dis. 2017;14(1):85-99. doi:10.1177/1479972316679664

20. Keating A, Lee A, Holland AE. What prevents people with chronic obstructive pulmonary disease from attending pulmonary rehabilitation? A systematic review. Chron Resp Dis. 2011;8(2):89-99. doi:10. $1177 / 1479972310393756$

21. Thorpe O, Johnston K, Kumar S. Barriers and enablers to physical activity participation in patients with COPD a systematic review. J Cardiopulm Rehabil Prev. 2012;32(6):359-369. doi:10.1097/HCR. 0b013e318262d7df

22. Cox NS, Oliveira CC, Lahham A, Holland AE. Pulmonary rehabilitation referral and participation are commonly influenced by environment, knowledge, and beliefs about consequences: a systematic review using the theoretical domains framework. $J$ Physiother. 2017;63(2):84-93. doi:10.1016/j.jphys.2017.02.002

23. Milner SC, Boruff JT, Beaurepaire C, Ahmed S, Janaudis-Ferreira T. Rate of, and barriers and enablers to, pulmonary rehabilitation referral in COPD: a systematic scoping review. Respir Med. 2018;13 7:103-114. doi:10.1016/j.rmed.2018.02.021

24. Brawley LR, Rejeski WJ, King AC. Promoting physical activity for older adults: the challenges for changing behavior. Am J Prev Med. 2003;25(3, Supplement 2):172-183. doi:10.1016/S0749-3797(03)00182-X

25. Jadczak AD, Dollard J, Mahajan N, Visvanathan R. The perspectives of pre-frail and frail older people on being advised about exercise: a qualitative study. Fam Pract. 2018;35(3):330-335. doi:10.1093/ fampra/cmx 108

26. Craig P, Dieppe P, Macintyre S, Michie S, Nazareth I, Petticrew M. Developing and evaluating complex interventions: the new Medical Research Council guidance. Brit Med J. 2008;337:a1655.

27. McLeroy KR, Bibeau D, Steckler A, Glanz K. An ecological perspective on health promotion programs. Health Educ Q. 1988;15 (4):351-377. doi:10.1177/109019818801500401

28. Fletcher A, Jamal F, Moore G, Evans RE, Murphy S, Bonell C. Realist complex intervention science: applying realist principles across all phases of the Medical Research Council framework for developing and evaluating complex interventions. Evaluation (Lond). 2016;22 (3):286-303. doi:10.1177/1356389016652743

29. Moore GF, Evans RE. What theory, for whom and in which context? Reflections on the application of theory in the development and evaluation of complex population health interventions. SSM Popul Health. 2017;3:132-135. doi:10.1016/j.ssmph.2016.12.005

30. Wong G, Greenhalgh T, Westhorp G, Buckingham J, Pawson R. RAMESES publication standards: realist syntheses. BMC Med. 2013; 11(1):21. doi:10.1186/1741-7015-11-21

31. Bunn F, Goodman C, Reece Jones P, et al. What works for whom in the management of diabetes in people living with dementia: a realist review. BMC Med. 2017;15(1):141. doi:10.1186/s12916-017-0909-2

32. Kastner M, Hayden L, Wong G, et al. Underlying mechanisms of complex interventions addressing the care of older adults with multimorbidity: a realist review. BMJ Open. 2019;9(4):e025009. doi:10.11 36/bmjopen-2018-025009
33. Pawson R, Greenhalgh T, Harvey G, Walshe K. Realist review a new method of systematic review designed for complex policy interventions. J Health Serv Res Policy. 2005;10(Suppl 1):21-34. doi:10.1258/1355819054308530

34. Wong G, Westhorp G, Pawson R, Greenhalgh T Realist synthesis: RAMESES training materials. 2013. Retrieved from: http://ramese sproject.org/media/Realist_reviews_training_materials.pdf. Accessed July 26, 2018.

35. Caspersen CJ, Powell KE, Christenson GM. Physical activity, exercise, and physical fitness: definitions and distinctions for health-related research. Public Health Rep. 1985;100(2):126-131.

36. Mirza S, Clay RD, Koslow MA, Scanlon PD. COPD guidelines: a review of the 2018 GOLD report. Mayo Clinic Proc. 2018;93 (10):1488-1502. doi:10.1016/j.mayocp.2018.05.026

37. National Institute for Health and Care Excellence. Diagnosing and assessing COPD. 2019. Retrieved from: https://pathways.nice.org.uk/ pathways/chronic-obstructive-pulmonary-disease\#path=view\%3A/ pathways/chronic-obstructive-pulmonary-disease/diagnosing-andassessing-COPD.xml\&content=view-info-category\%3Aview-aboutmenu. Accessed January 8, 2019.

38. Fried LP, Tangen CM, Walston J, et al. Frailty in older adults: evidence for a phenotype. J Gerontol a Biol Sci Med Sci. 2001;56 (3):M146-M156. doi:10.1093/gerona/56.3.M146

39. Guralnik JM, Simonsick EM, Ferrucci L, et al. A short physical performance battery assessing lower extremity function: association with self-reported disability and prediction of mortality and nursing home admission. J Gerontol. 1994;49(2):M85-M94. doi:10.1093/geronj/49.2.M85

40. European Medicines Agency. Reflection paper on physical frailty: instruments for baseline characterisation of older populations in clinical trials. 2018. Retrieved from: https://www.ema.europa. eu/documents/scientific-guideline/reflection-paper-physical-frailtyinstruments-baseline-characterisation-older-populations-clinical_en. pdf. Accsessed October 02, 2018.

41. Rockwood K, Song X, MacKnight C, et al. A global clinical measure of fitness and frailty in elderly people. CMAJ. 2005;173(5):489-495. doi:10.1503/cmaj.050051

42. Paulson D, Lichtenberg PA. The paulson-lichtenberg frailty index: evidence for a self-report measure of frailty. Aging Ment Health. 2015;19(10):892-901. doi:10.1080/13607863.2014.986645

43. Greenhalgh T, Macfarlane F, Steed L, Walton R. What works for whom in pharmacist-led smoking cessation support: realist review. BMC Med. 2016;14(1):209. doi:10.1186/s12916-016-0749-5

44. Schreuders M, Nuyts PAW, van den Putte B, Kunst AE. Understanding the impact of school tobacco policies on adolescent smoking behaviour: a realist review. Soc Sci Med. 2017;183:19-27. doi:10.1016/j.socscimed.2017.04.031

45. Torres-Sanchez I, Valenza MC, Cabrera-Martos I, Lopez-Torres I, Benitez-Feliponi A, Conde-Valero A. Effects of an exercise intervention in frail older patients with chronic obstructive pulmonary disease hospitalized due to an exacerbation: a randomized controlled trial. COPD. 2017;14(1):37-42. doi:10.1080/15412555.2016.1209476

46. Vivodtzev I, Pépin J-L, Vottero G, et al. Improvement in quadriceps strength and dyspnea in daily tasks after 1 month of electrical stimulation in severely deconditioned and malnourished COPD. Chest. 2006;129(6):1540-1548. doi:10.1378/chest.129.6.1540

47. Boxall A-M, Barclay L, Sayers A, Caplan GA. Managing chronic obstructive pulmonary disease in the community: a randomized controlled trial of home-based pulmonary rehabilitation for elderly housebound patients. J Cardiopulm Rehabil Prev. 2005;25(6):37 8-385. doi:10.1097/00008483-200511000-00012

48. Wedzicha JA, Bestall JC, Garrod R, Garnham R, Paul EA, Jones PW. Randomized controlled trial of pulmonary rehabilitation in severe chronic obstructive pulmonary disease patients, stratified with the MRC dyspnoea scale. Eur Respir J. 1998;12(2):363-369. doi:10.11 $83 / 09031936.98 .12020363$ 
49. Kennedy C, Novotny P, LeBrasseur N, Wise R, Sciurba F, Benzo R. Does pulmonary rehabilitation impact frailty? Am J Transplant. 2015;15(Supplement):3. doi:10.1111/ajt.13045

50. Kennedy C, Novotny P, Stevens E, et al. Prospective trial using pulmonary rehabilitation to treat frailty. Paper presented at: 2018 American Transplant Congress; 2018; Seattle, WA, USA.

51. McClellan R, Amiri HM, Limsuwat C, Nugent KM. Pulmonary rehabilitation increases gait speed in patients with chronic lung diseases. Health Serv Res Managerial Epidemiol. 2014;1:23333928 14533659. doi:10.1177/2333392814533659

52. Mittal N, Raj R, Islam E, Nugent K. Pulmonary rehabilitation improves frailty and gait speed in some ambulatory patients with chronic lung diseases. Southwest Res Crit Care Chronicals. 2015;3 (12):2-10.

53. Plas J, Ter Beek L, van der Vaart H, Jager-Wittenaar H. Improvement in frailty status after pulmonary rehabilitation in patients with chronic obstructive pulmonary disease. Clin Nutr. 2019;38(Supplement 1): S116-S117. doi:10.1016/S0261-5614(19)32787-6

54. Sullivan KO, Mulkerns A, Toole PO, McDonnell TJ. Does pulmonary rehabilitation reduce frailty in patients with COPD? Irish $J$ Med Sci. 2016;185:S464-S464.

55. Blindenbach S, Vrancken JWFA, van der Zeijden H, et al. Effects of geriatric COPD rehabilitation on hospital admissions and exercise tolerance: a retrospective observational study. Tijdschr Gerontol Geriatr. 2017;48(3):112-120. doi:10.1007/s12439-017-0214-8

56. van Dam van Isselt EF, Spruit $\mathrm{M}$, Groenewegen-Sipkema KH, Chavannes NH, Achterberg WP. Geriatric rehabilitation for patients with advanced chronic obstructive pulmonary disease: a naturalistic prospective cohort study on feasibility and course of health status. Chron Resp Dis. 2014;11(2):111-119. doi:10.1177/1479972314529674

57. van Dam van Isselt $\mathrm{EF}$, van Eijk $\mathrm{M}$, van Geloven $\mathrm{N}$, et al. A prospective cohort study on the effects of geriatric rehabilitation following acute exacerbations of COPD. J Am Med Dir Assoc. 2019;20(7):850-856.e2. doi:10.1016/j.jamda.2019.02.025

58. Lizak MK, Singh S, Lubina S, Zembala M. Female and male chronic obstructive pulmonary disease patients with severe dyspnea do not profit less from pulmonary rehabilitation. Pol Arch Med Wewn. 2008;118(7-8):413-418.

59. Osadnik C, Loeckx M, Louvaris Z, et al. The likelihood of improving physical activity after pulmonary rehabilitation is increased in patients with COPD who have better exercise tolerance. Int J Chron Obstruct Pulmon Dis. 2018;13:3515-3527. doi:10.2147/COPD.S174827

60. Benzo R, Wetzstein M, Neuenfeldt P, McEvoy C. Implementation of physical activity programs after COPD hospitalizations: lessons from a randomized study. Chron Resp Dis. 2015;12(1):5-10. doi:10.1177/ 1479972314562208

61. Brighton LJ, Bristowe K, Bayly J, et al. Experiences of pulmonary rehabilitation in people living with COPD and frailty: a qualitative interview study. Under Review.

62. Harrison SL, Robertson N, Apps L, Steiner MC, Morgan MDL, Singh SJ. "We are not worthy" - understanding why patients decline pulmonary rehabilitation following an acute exacerbation of COPD. Disabil Rehabil. 2015;37(9):750-756. doi:10.3109/09638288.2014. 939770

63. Mathar H, Fastholm P, Lange P, Larsen NS. Why do patients decline participation in offered pulmonary rehabilitation? A qualitative study. Clin Rehabil. 2017;31(12):1674-1683. doi:10.1177/0269215517708 821

64. van Dam van Isselt EF, Spruit-van Eijk M, Chavannes NH, Achterberg WP, Groenewegen-Sipkema KH. Geriatric rehabilitation for patients with advanced COPD: programme characteristics and case studies. Int J Palliat Nurs. 2013;19(3):141-146. doi:10.12968/ ijpn.2013.19.3.141

65. Celis-Preciado CA, Borda M, Castelblanco S, et al. Sarcopenia and frailty, two new domains in chronic obstructive pulmonary disease prognosis: a systematic review. Am J Resp Crit Care. 2017;195.
66. Cesari M, Costanzo L, Giua R, Vellas B, Incalzi RA. Physical function and exercise in older patients with cardiovascular and respiratory conditions. Curr Pharm Des. 2014;20(19):3198-3214. doi:10.2174/ 13816128113196660691

67. Attwell L, Vassallo M. Response to pulmonary rehabilitation in older people with physical frailty, sarcopenia and chronic lung disease. Geriatrics. 2017;2(1):9. doi:10.3390/geriatrics2010009

68. Crisafulli E, Morandi A, Olivini A, Malerba M, Clini EM. Rehabilitation and supportive therapy in elderly patients with chronic obstructive pulmonary disease. Eur J Intern Med. 2014;25(4):32 9-335. doi:10.1016/j.ejim.2014.01.003

69. Holland AE, Harrison SL, Brooks D. Multimorbidity, frailty and chronic obstructive pulmonary disease: are the challenges for pulmonary rehabilitation in the name? Chron Resp Dis. 2016;13 (4):372-382. doi:10.1177/1479972316670104

70. Guan C, Niu H. Frailty assessment in older adults with chronic obstructive respiratory diseases. Clin Interv Aging. 2018;13:1513-15 24. doi:10.2147/CIA.S173239

71. Benton MJ, Swan PD. Addition of resistance training to pulmonary rehabilitation programs: an evidence-based rationale and guidelines for use of resistance training with elderly patients with COPD. Cardiopulm Phys Ther J. 2006;17(4):127-133. doi:10.1097/01823 246-200617040-00002

72. Spruit MA, Wouters EFM. Organizational aspects of pulmonary rehabilitation in chronic respiratory diseases. Respirology. 2019. doi:10.1111/resp. 13512

73. Wedzicha JA. Rehabilitation in severe chronic obstructive pulmonary disease. Lancet. 1996;348(9042):1661. doi:10.1016/S0140-6736(05) 65728-5

74. Charbek E, Espiritu JR, Nayak R, Morley JE. Frailty, comorbidity, and COPD. J Nutr Health Aging. 2018;22(8):876-879. doi:10.1007/ s12603-018-1068-7

75. Dyer C, Pugh L. Lung health in older adults. Age \& Ageing. 2019;48 (3):319-322. doi:10.1093/ageing/afz008

76. Dent E, Martin FC, Bergman H, Woo J, Romero-Ortuno R, Walston JD. Management of frailty: opportunities, challenges, and future directions. Lancet. 2019;394(10206):1376-1386. doi:10.1016/ S0140-6736(19)31785-4

77. Troosters T, Blondeel A, Janssens W, Demeyer H. The past, present and future of pulmonary rehabilitation. Respirology. 2019;24(9):8 30-837. doi:10.1111/resp.v24.9

78. McNamara RJ, Dale M, McKeough ZJ. Innovative strategies to improve the reach and engagement in pulmonary rehabilitation. $J$ Thorac Dis. 2019;11(S17):S2192-S2199. doi:10.2 $1037 /$ jtd

79. Thiyagarajan JA, Araujo de Carvalho I, Pena-Rosas JP, et al. Redesigning care for older people to preserve physical and mental capacity: WHO guidelines on community-level interventions in integrated care. PLoS Med. 2019;16(10):e1002948. doi:10.1371/journal. pmed. 1002948

80. Liang J, Abramson MJ, Russell G, et al. Interdisciplinary COPD intervention in primary care: a cluster randomised controlled trial. Eur Respir J. 2019;53(4):1801530. doi:10.1183/13993003.015302018

81. Brighton LJ, Miller S, Farquhar M, et al. Holistic services for people with advanced disease and chronic breathlessness: a systematic review and meta-analysis. Thorax. 2019;74(3):270-281. doi:10.11 36/thoraxjnl-2018-211589

82. Jones AV, Evans RA, Esliger DW, Sherar LB, Singh SJ. Protocol for a feasibility trial to inform the development of a breathlessness rehabilitation programme for chronic obstructive pulmonary disease and chronic heart failure (the COHERE trial). BMJ Open. 2019;9(7):e029387. doi:10.1136/bmjopen-2019-029387

83. Moore GF, Audrey S, Barker M, et al. Process evaluation of complex interventions: Medical Research Council guidance. BMJ. 2015;350 (mar19 6):h1258. doi:10.1136/bmj.h1258 
84. Steiner M, McMillan V, Lowe D, et al. Pulmonary Rehabilitation: An Exercise in Improvement. National Chronic Obstructive Pulmonary Disease (COPD) Audit Programme: Clinical and Organisational Audits of Pulmonary Rehabilitation Services in England and Wales 2017. London: Royal College of Physicians; 2018.
85. Nolan CM, Kaliaraju D, Jones SE, et al. Home versus outpatient pulmonary rehabilitation in COPD: a propensity-matched cohort study. Thorax. 2019;74(10):996-998. doi:10.1136/thoraxjnl-2018-212765

\section{Publish your work in this journal}

The International Journal of COPD is an international, peer-reviewed journal of therapeutics and pharmacology focusing on concise rapid reporting of clinical studies and reviews in COPD. Special focus is given to the pathophysiological processes underlying the disease, intervention programs, patient focused education, and self management protocols. This journal is indexed on PubMed Central, MedLine and CAS. The manuscript management system is completely online and includes a very quick and fair peer-review system, which is all easy to use. Visit http://www.dovepress.com/testimonials.php to read real quotes from published authors. 